\title{
Studies of Electrospun Cellulose Acetate Nanofibrous Membranes
}

\author{
Weitao Zhou ${ }^{1,2}$, Jianxin $\mathrm{He}^{2}$, Shizhong Cui ${ }^{*}, 1,2$ and Weidong Gao ${ }^{1}$ \\ ${ }^{I}$ School of Textile \& Clothing, Jiangnan University, Wuxi-214122, P.R. China \\ ${ }^{2}$ School of Textiles, Zhongyuan University of Technology, Zhengzhou-450007, P.R. China
}

\begin{abstract}
The objective of this work was to study the feasibility of preparing cellulose acetate (CA) nanofibrous membranes by electrospinning with single solvent and their potential applying as affinity membrane. CA solutions in dichloromethane (DCM), formic acid, acetic acid and trifluoroacetic acid (TFA) were electrospun into nonwoven fiber membranes, respectively. The scanning electron microscope (SEM) results showed that the as-spun CA nanofibers using TFA as solvent were continuous and smooth with the fiber diameter ranging from 100-300nm. Furthermore, their properties such as specific surface area, porosity and mechanical properties were investigated. The nanofibrous membranes exhibited high surface area and high porosity compared with common filter paper, which reached $2.020 \times 10^{7} \mathrm{~m}^{-1}$ and $87 \%$, respectively. Also, the membranes displayed a very high water permeability and a good hydrolytic stability. These characteristic properties were very effective for the separation of particles. As a result of X-ray diffraction (XRD), the obtained fibers were mainly amorphous, but characterized for good mechanical properties.
\end{abstract}

Keywords: Cellulose acetate, electrospinning, morphology, porosity, mechanical properties.

\section{INTRODUCTION}

Since mid-1990s, many research groups in materials and biomedical fields have been of interest in electrospinning techniques and electrospun nanofibers. With a wide variety of materials, electrospinning techniques and theoretic have been dramatically developed, as highlighted by Yang DY et al. [1], Cao $\mathrm{H}$ et al. [2], Thompson CJ et al. [3] and Xu L [4]. Nowadays, the main focus of the electrospinning study moves to the electrospinning of the blend materials, the solvent system and the applications of the electrospun materials in various fields, such as high-performance air filters reported by Opal $\mathrm{R}$ et al. [5], heavy metal ions adsorbent reported by Ki CS [6], sensor studied by Wang XF et al. [7] protective textile stated by Schreuder-Gibson HL et al. [8], wound dressing found by Nakagawa Y [9], and as scaffolds in tissue engineering reported by Vepari C \& Kaplan DL [10]. Filter application of the electrospun material is considered as a special interest for industrialization. Recently, water permeable nanofibrous membrane is discussed widely.

Cellulose acetate (CA), a derivative of cellulose, can be easily obtained from natural resources and recycled in environment by biodegradation. According to Chronakis $\mathrm{J}$ [11], it can be easily fabricated into films, membranes and fibers. CA has been widely used in membranes for separation and medical application, because of its good hydrolytic stability and relatively low cost. Electrospinning is known to be a simple and versatile method for the production of nanofibers. Nowadays, CA nanofibers had been prepared by using binary or ternary solvent system. Liu HQ \& Hesieh YL [12] investigated the relationship between

\footnotetext{
*Address correspondence to this author at the School of Textiles, Zhongyuan University of Technology, Zhengzhou, 45007, China; Tel: +86371-69975729; Fax: +86-371-62506970; E-mail: snowballer@163.com
}

CA solution properties and morphology of nanofibers, included that a mixture of acetone/dimethylacet amide (2:1) was good for CA electrospinning with the fiber diameter ranging from $1-2 \mu \mathrm{m}$. CA solution in a mixture of acetone/DMF/trifluoroethylene (3:1:1) was electrospun into nonwoven fiber mesh with the fiber diameter ranging from $200 \mathrm{~nm}$ to $1 \mu \mathrm{m}$ reported by Ma Z et al. [13]. Han SO et al. [14] prepared ultrafine porous cellulose triacetate fibers by electrospinning with methylene chloride and a mixed solvent of methylene chloride/ethanol.

However, these binary or ternary solvent systems go against blending spinning of CA. In this study, CA was dissolved in four common solvents such as dichloromethane (DCM), formic acid, acetic acid and trifluoroacetic acid (TFA), respectively, and then were electrospun to fibers. The effects of solvents on spinnability of electrospun CA nanofiber and the performances of the as-spun nanofibrous membranes were investigated.

\section{EXPERIMENTAL}

\subsection{Materials}

CA (white powder; polymerization degree $\sim 200$; degree of acetyl substitution $\sim 2.5$ ) was provided by Rayonier Inc (China), and the chemical structure was shown in Fig. (1). All the reagents were of analytical reagent grade and used without further purification. Some properties of interest for these solvents (i.e. boiling point, melting point, density, conductivity, viscosity) were summarized in Table $\mathbf{1}$ according to Cheng NL \& Hu NS [15].

\subsection{Membrane Fabrication}

For the electrospinning, CA powder was dissolved in DCM, 98\% formic acid, acetic acid and TFA at room temperature for $4 \mathrm{~h}$, respectively. The solutions for electrospinning were homogeneous without any precipitates and air bubbles. The mass fraction of the CA spinning 
solution was $8 \%$. Before electrospinning, CA solutions were characterized for their viscosity and conductivity using NDJ79 rotary viscosimeter and DDS-11A conductivity meter at room temperature, respectively. Then, each spinning solution was placed in a $10 \mathrm{ml}$ syringe with a stainless steel syringe needle (inner diameter of $0.7 \mathrm{~mm}$ ) as an electrode which connected to direct current (DC) high-voltage generator. The syringe was loaded in a syringe pump to control a flow rate accurately. A piece of aluminum foil was used as a collector for obtaining sheet type nanofiber assemblies and grounded. The electrospinning experiments were performed at room temperature and $60 \%$ humidity. The flow rate of spinning solution was controlled at $0.3 \mathrm{ml} / \mathrm{h}$. Electric potential and distance to collector were fixed at $15 \mathrm{kV}$ and $100 \mathrm{~mm}$, respectively.

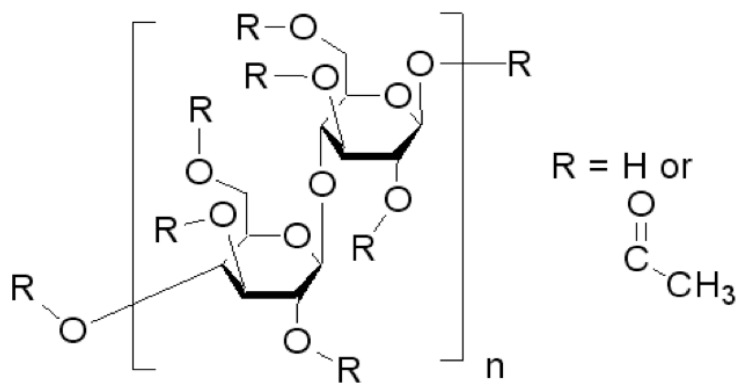

Fig. (1). Chemical structure of CA.

(a)

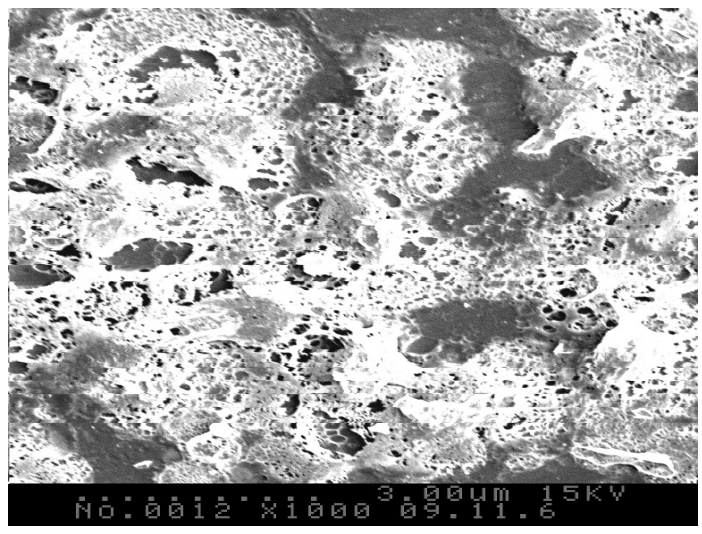

(c)

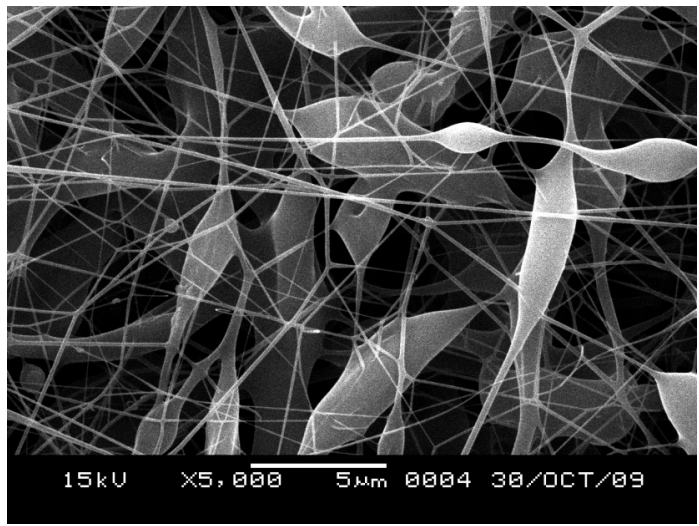

\subsection{Membrane Characterization}

Morphological structure of the electrospun CA nanofibers with different solvents were observed using scanning electron microscope (SEM) (JSM-6360LVSEM, JEOL Co., Japan) after gold coating and the diameter of the nanofiber were measured by an image processing software. A specific surface area of the membrane was obtained by the following equation:

$S=\frac{A}{V}$.

where $A$ was the total surface area of the nanofiber and $V$ was the volume. Assuming that the nanofiber was a cylinder, $A$ and $V$ could be calculated from measured mass and density of the membrane by equation (2) and (3), respectively:

$A=\pi D L=\frac{4 m}{\rho D}$

$V=\frac{m}{\rho}=\pi\left(\frac{D}{2}\right)^{2} L$

where $m$ was the mass of the membrane and $\pi$ was the density of the nanofiber assuming the nanofiber was rigid without pore or void. $D$ was a mean diameter and $L$ was total length of the nanofiber. The fiber density, $\rho$, was obtained by measuring the mass of the membrane in the air and in liquid (n-butyl alcohol) using the following equation:

(b)

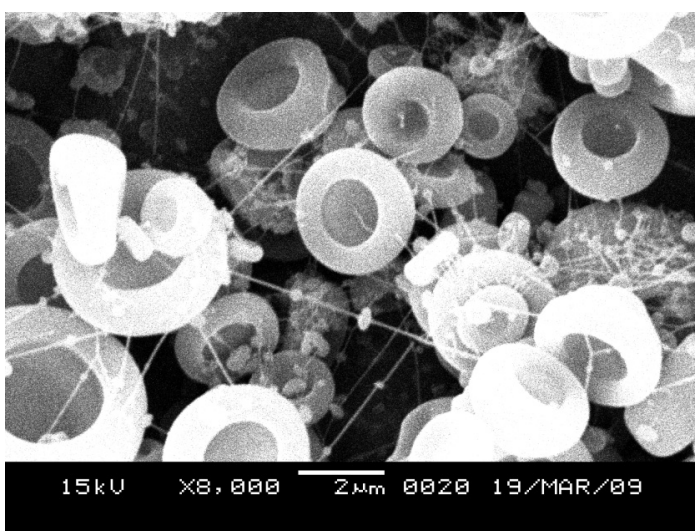

(d)

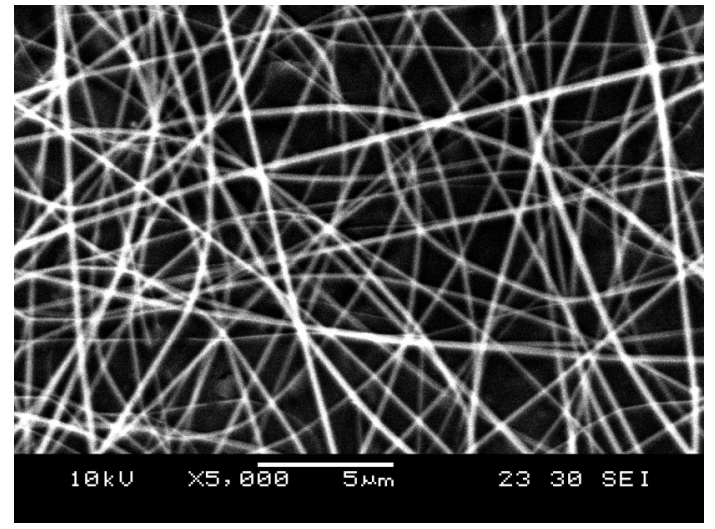

Fig. (2). Morphological structures of the electrospun CA fibers with different solvents, (a) DCM, (b) 98\% formic acid, (c) acetic acid and (d) TFA. 
Table1. Properties of the Solvents Used in this Work

\begin{tabular}{|c|c|c|c|c|c|}
\hline Solvent & $\begin{array}{c}\text { Boiling Point } \\
/{ }^{\circ} \mathbf{C}\end{array}$ & $\begin{array}{c}\text { Melting Point } \\
/{ }^{\circ} \mathbf{C}\end{array}$ & $\begin{array}{c}\text { Density } \\
/ \mathbf{g} \cdot \mathbf{c m}^{-3}\end{array}$ & $\begin{array}{c}\text { Conductivity } \\
\mathbf{S} \cdot \mathbf{m}^{-1}\end{array}$ & $\begin{array}{c}\text { Viscosity } \\
/ \mathbf{m P a} \cdot \mathbf{s}\end{array}$ \\
\hline \hline DCM & 39.8 & -95.1 & 1.30 & $4.9 \times 10^{-9}$ & 0.42 \\
Formic acid & 100.8 & 8.4 & 1.22 & $6.4 \times 10^{-5}$ & 1.57 \\
Acetic acid & 117.9 & 16.6 & 1.05 & $1.12 \times 10^{-8}$ & 1.3 \\
TFA & 72.4 & -15.6 & 1.53 & $2.6 \times 10^{5}$ & 0.81 \\
\hline
\end{tabular}

Note: Boiling point test in $1.0 \mathrm{Mpa}$ density test at $25^{\circ} \mathrm{C}$.

$\rho=\frac{m_{1}}{m-m_{2}} \rho_{b}$

where $m_{1}$ was the mass of the membrane in the air and $m_{2}$ was the mass of the membrane in n-butyl alcohol. And $\rho_{b}$ was a density of n-butyl alcohol at measured temperature.

The porosity $(\varepsilon)$ of the membrane can be calculated by measuring the volume of the membrane and the nanofiber volume by the following equation:

$\varepsilon=\frac{V_{m}-V}{V_{m}}=1-\frac{V}{V_{m}}=1-\frac{V}{A_{m} t}$

where $V_{m}$ was total volume of the membrane which could be obtained by measuring the area $\left(A_{m}\right)$ and thickness $(t)$ of membrane. The thickness was measured by using a micrometer caliper.

Fourier transform infrared (FTIR) spectra were obtained using a FTIR spectrophotometer (NEXUS670, America) in the spectral region of $400-4000 \mathrm{~cm}^{-1}$, the powdered electrospun $\mathrm{CA}$ nanofibers were pressed into potassium bromide $(\mathrm{KBr})$ pellets prior to data collection.

X-ray diffraction (D/max-2550PC, Rigaku Corporation, Japan) was operated at $40 \mathrm{kV}$ tube voltage and $40 \mathrm{~mA}$ tube current, $\mathrm{CuK} \alpha$ radiation was used with diffraction angle $2 \theta$ from $2^{\circ}$ to $60^{\circ}$, the scanning rate was $2^{\circ} / \mathrm{min}$ with powdered electrospun CA fibers.

For mechanical strength test, the nanofibrous membranes were cut into strips with a dimension of $1 \mathrm{~cm} \times 4 \mathrm{~cm}$, and then mounted on an Instron 5848 to test the tensile stress curve at a stretching speed of $10 \mathrm{~mm} / \mathrm{min}$. The mesh thickness measured by a micrometer and the sample width $(1 \mathrm{~cm})$ was input into the computer to calculate the tensile stress.

\section{RESULTS AND DISSCUSSION}

\subsection{Morphological Characteristics}

Fig. (2) showed the morphological structures of the electrospun CA products with different solvents (DCM, 98\% formic acid, acetic acid and TFA), and some of properties, i.e. viscosity and conductivity of the $\mathrm{CA}$ solutions were summarized in Table 2. Electrospinning of CA solution in DCM was impossible. The cast film with adhesion and holes was obtained (Fig. 2a), because the extremely low conductivity and high viscosity of the spinning solution (Table 2) caused the spinneret spinning droplet to stretch ineffectively. Few fine fibers and many strange "bellshaped" particles were obtained when formic acid was used as solvent (Fig. 2b). It might be caused by the poor conductivity of the solution, which was consistent with the results found by Han SO [14]. The solution of CA in acetic acid produced a large amount of fibers with very fine diameter and some slubs or spindles, because the relatively high boiling point $\left(117.9^{\circ} \mathrm{C}\right)$ of acetic acid caused the solvent difficult to evaporate during the drafting process. In comparison with CA solutions in DCM, formic acid and acetic acid, the electrospun CA nanofibers using TFA as solvent were continuous and smooth without any beads or droplets in the surface (Fig. 2d), and their diameter distributions were relatively concentrated with the fiber diameter ranging from $100-300 \mathrm{~nm}$ (Fig. 3), indicating excellent electro-spinnability of the CA solutions in TFA solvent. It might be caused by the low boiling point $\left(72.4^{\circ} \mathrm{C}\right)$ and high conductivity (TFA was a strong acid, could ionize out a lot of $\mathrm{H}^{+}$and $\mathrm{F}^{-}$) of TFA solvent.

Table 2. Viscosity and Conductivity of CA Solutions in DCM, Formic Acid, Acetic Acid and TFA

\begin{tabular}{|c|c|c|}
\hline Solvent & Viscosity $/ \mathbf{P a} \cdot \mathbf{s}$ & Conductivity $/ \mathbf{S} \cdot \mathbf{m}^{-1}$ \\
\hline \hline DCM & 22.31 & $3.12 \times 10^{-5}$ \\
Formic acid & 3.07 & $3.93 \times 10^{-5}$ \\
Acetic acid & 3.40 & $1.04 \times 10^{-4}$ \\
TFA & 5.29 & $9.62 \times 10^{-4}$ \\
\hline
\end{tabular}

\subsection{Membrane Properties}

The dimensions of CA nanofibrous membranes were listed in Table 3. The mean diameter of nanofibers was 0.198 $\mu \mathrm{m}$ and the thickness of membranes was $406 \mu \mathrm{m}$. Also, the specific surface area and porosity were calculated and listed in Table 3. The specific surface area of nanofiber membrane was approximately $2.020 \times 10^{7} \mathrm{~m}^{-1}$ and the porosity was around $87 \%$. As compared with the filter paper, the specific area of CA Nanofibrous membranes increased by 80 times and the porosity was improved almost 2 times. Since the electrospun Nanofibrous membrane had very large surface area, this characteristic property was very effective for the adsorption of ions, particles and became an advantage for affinity membrane which separated specific molecules through adsorption based on physical or chemical affinity. The porosity was also an important parameter for the membrane. Though the Nanofibrous membrane had a compact structure, it was an open pore structure and therefore, highly porous structure (more than $80 \%$ porosity) helped easy permeation of liquid.

In order to measure the permeability of the membrane, circular shape of $47 \mathrm{~mm}$ diameter was stamped out from the electrospun nanofibrous membrane and placed in filterholder. $200 \mathrm{ml}$ distilled water was pumped and recycled 
Table 3. Specific Surface Area and Porosity of CA Nanofibrous Membrane

\begin{tabular}{|c|c|c|c|c|}
\hline Membranes & Fiber Diameter/ $\mu \mathrm{m}$ & Thickness/ $/ \mu \mathrm{m}$ & Specific Surface Area $/ \times 10^{7} \mathrm{~m}^{-1}$ & Porosity $/ \%$ \\
\hline $\mathrm{CA}$ & $0.198 \pm 0.05$ & 406 & 2.020 & 87 \\
\hline Filter paper & $16.13 \pm 3.74$ & 79 & 0.025 & 48 \\
\hline
\end{tabular}

through the nanofiber membrane with a peristaltic pump (BT100-2J, Longer Pump Corporation, China). The volumetric flow rate was gradually increased from 1$100 \mathrm{ml} / \mathrm{min}$. The results showed the membranes exhibited a very high water permeability. When the volumetric flow rate was $10 \mathrm{ml} / \mathrm{min}$, the distilled water could easily permeate. The volumetric flow rate steadily increased from 10 to $60 \mathrm{ml} / \mathrm{min}$ and the water flowed smoothly. But when the volumetric flow rate reached $70 \mathrm{ml} / \mathrm{min}$, the water permeation became difficult. Moreover, the morphology variations after the testing was observed with a JEM-2100F Field Emission SEM. Field emission electron microscope (FEEM) images were taken to study the morphological changes of the CA nanofibrous membrane following permeability test. As shown in Fig. (4), no obvious changes of morphology in the CA nanofibrous membrane were observed after permeability test, indicating the CA nanofibrous membranes had good hydrolytic stability.

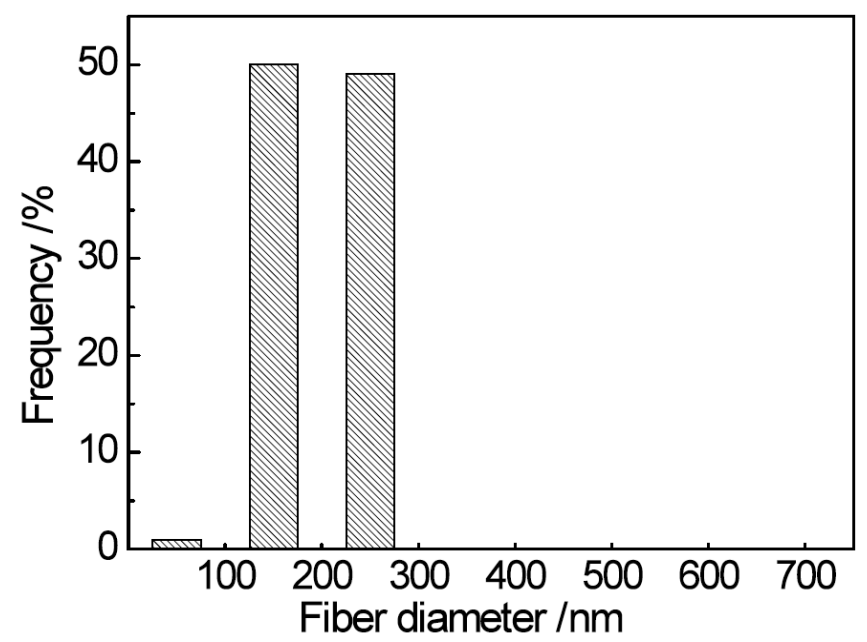

Fig. (3). Diameter distributions of electrospun CA nanofibers with TFA as solvent.

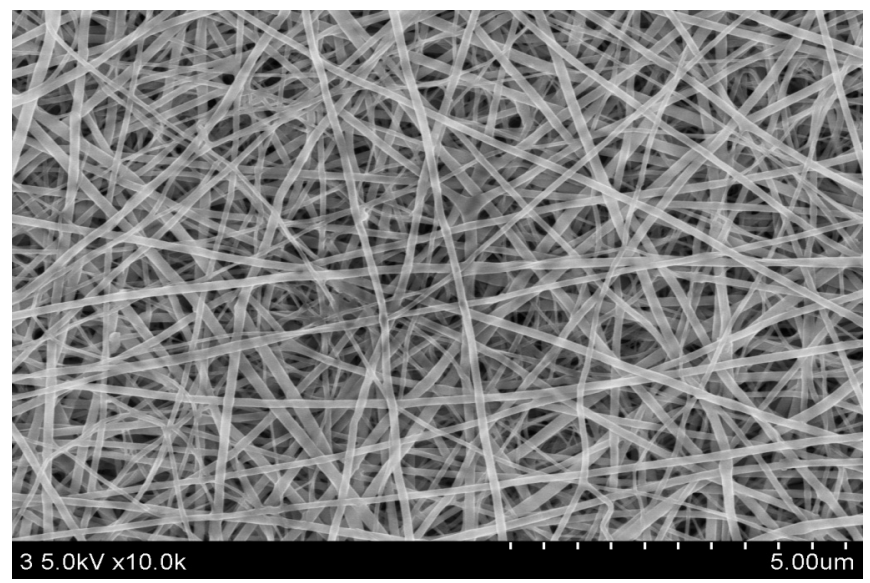

Fig. (4). FEEM micrograph of CA nanofibrous membrane after permeability test.

\subsection{FTIR Spectra}

The IR spectrum of CA nanofibrous membrane was shown in Fig. (5). CA membrane showed two strong adsorption bands at $1752 \mathrm{~cm}^{-1}$ and $1236 \mathrm{~cm}^{-1}$, which attributed to the $\mathrm{C}=\mathrm{O}$ stretching and the acetyl groups, respectively. According to He Jianxin [16] the adsorption band at $1635 \mathrm{~cm}^{-1}$ was assigned to the water adsorption, and $1371 \mathrm{~cm}^{-1}$ and $1434 \mathrm{~cm}^{-1}$ were assigned to the symmetric and asymmetric vibrations of $\mathrm{CH}_{3}$.

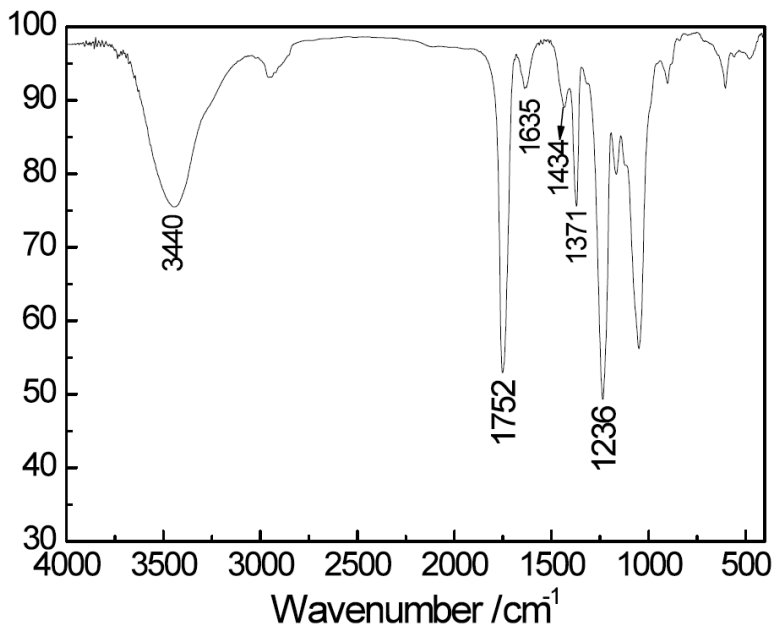

Fig. (5). IR spectra of electrospun CA nanofibers.

\subsection{Crystalline Structure and Mechanical Properties}

Fig. (6) showed the XRD curve of CA nanofibers. Two broad peaks appeared at $2 \theta 10.2^{\circ}$ and $21.7^{\circ}$, indicating that the structure of the as-spun CA nanofibers was amorphous, which was consistent with He Jianxin et al. [16]. CA solution in a mixture of acetone/DMF/ trifluoroethylene (3:1:1) was electrospun into nonwoven fiber mesh with the

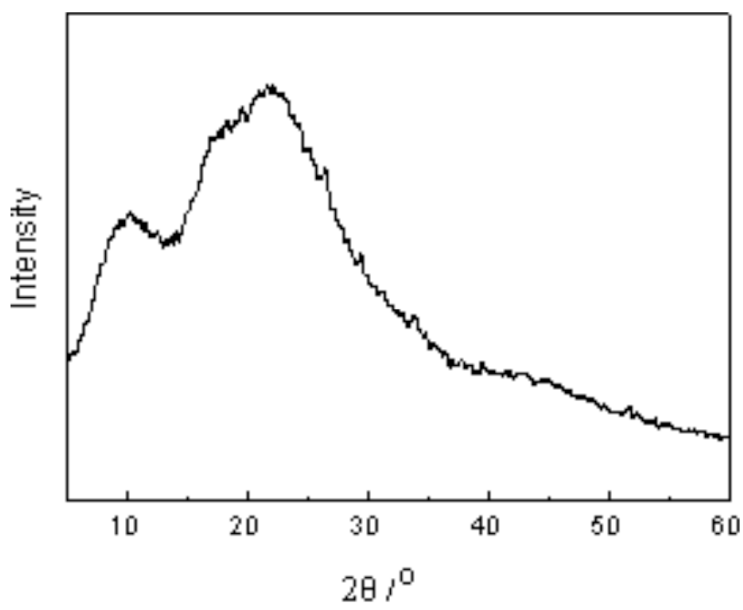

Fig. (6). XRD image of electrospun CA nanofibrous membrane. 
fiber diameter ranging from $200 \mathrm{~nm}$ to $1 \mu \mathrm{m}$ reported by Zuwei Ma et al. [13], and the CA nanofiber membrane displayed a poor tensile strength (about $100 \mathrm{cN} / \mathrm{mm}^{2}$ ) and a high elongation ratio $(40 \%)$ at break. The tensile stress curve of the as-spun CA membranes was shown in Fig. (7). In comparison with the mechanical properties of CA nanofiber mats reported by Zuwei Ma et al. [13], the tensile strength of CA nanofiber mats was much higher, which reached $485.9 \mathrm{cN} / \mathrm{mm}^{2}$, but the breaking elongation decreased to $13.97 \%$. It might be due to its finer fiber diameter and more centralized diameter distribution, as the fiber diameter was reduced, the alignment of the molecules in the direction of fiber axis was improved, and the orientation of macro molecules existing in the super molecular structures of the amorphous phase played a dominant role in increasing the fiber mechanical properties as reported by Wong SC et al. [17], Arinstein A et al. [18].

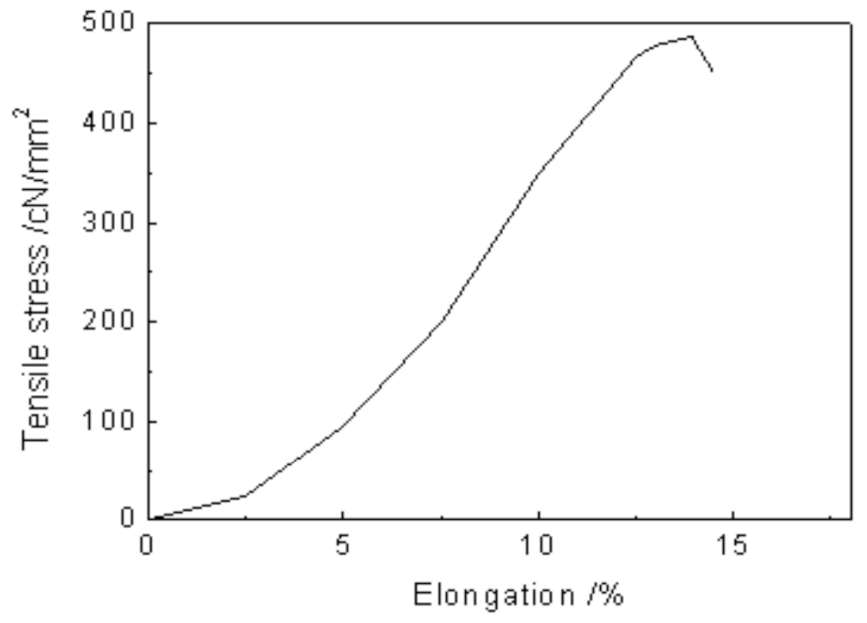

Fig. (7). Tensile stress curve of electrospun CA nanofibrous membrane.

\section{CONCLUSIONS}

$8 \%$ CA spinning solution with trifluoroacetic acid as solvent had good spinnability. Specifically, the fibers were continuous and smooth without any beads or droplets in the surface of the electrospun membrane, and the diameter ranged from 100 $300 \mathrm{~nm}$. Compared with common filter paper, the nanofibrous membranes exhibited high surface area and high porosity, which reached $2.020 \times 10^{7} \mathrm{~m}^{-1}$ and $87 \%$, respectively. Also, the membranes exhibited very high water permeability and hydrolytic stability. As a result of XRD, the obtained fibers were mainly amorphous, but characterizing for good mechanical properties. Electrospinning of CA nanofibrous membranes gave the possibility of producing new materials for potential filter.

\section{ACKNOWLEDGEMENTS} molecular structure on polymer nanofiber mechanical properties. Nat Nanotechnol 2007; 2: 59-62.
[18] Arinstein A, Burman M, Gendelman O, Zussman E. The effect of super

This research was financially supported by China Postdoctoral Science Foundation funded project (20080430079), and we also acknowledged the support from the Program of Introducing Talents of Discipline to Universities, B07024, China.

\section{REFERENCES}

[1] Yang DY, Liu XL, Jin Y, Zhu Y, Zeng DD, Jing XY, Ma HW. Electrospinning of poly (dimethylsiloxane) nanofibrous membrane: fabrication and application in protein microarrays. Biomacromolecules 2009; 10(12):3335-40.

[2] Cao H, Chen X, Huang L, Shao ZZ. Electrospinning of reconstituted silk fiber from aqueous silk fibroin solution. Mater Sci Eng C 2009; 29(7):2270-74.

[3] Thompson CJ, Chase GG, Yarin AL, DH. Effect of parameters on nanofiber diameter determined from electrospinning model. Polymer 2007; 48(23): 6913-22.

[4] Xu L. A mathematical model for electrospinning process under coupled field forces. Chaos Soliton Fractal.2009; 42(3): 1463-65.

[5] Opal R, Kaur S, Ma Z, Chan C, Ramskrishna S, Matsuurd T. Electrospun nanofibrous filtration membrane. J Membr Sci 2007; 281(2):581-6.

[6] Ki CS, Gang EH, Um IC, Park YH. Nanofibrous membrane of wool keratose/silk fibroin blend for heavy metal ion adsorption. J Membr Sci 2007; 302:20-26.

[7] Wang XF, Ding B, Sun M, Yu JY, Sun G. Nanofibrous polyethyleneimine membranes as sensitive coatings for quartz crystal microbalance-based formaldehyde sensors. Sensor Actuator B-Chem 2010; 144(1): 11-17.

[8] Schreuder-Gibson HL, Gibson P, Senecal K, et al. Protective textile materials based on electrospun nanofibers. J Adv Mater 2002; 34(3): 44-55.

[9] Nakagawa Y, Murai T, Hasegawa C, et al. Endotoxin contamination in wound dressings made of natural biomaterials. J Biomed Mater Res Part B 2003; 66B:347-55.

[10] Vepari C, Kaplan DL. Silk as a biomaterial. Prog Polym Sci 2007; 32:991-1007.

[11] Chronakis J. Novel nanocomposites and nanoceramics based on polymer nanofibers using electrospinning process-A review. J Mater Sci Technol 2005; 167(2-3):283-293.

[12] Liu HQ, Hesieh YL. Ultrafine fibrous cellulose membranes from electrospinning of cellulose acetate. J Polym Sci B-Polym Phys 2002; 40(18): 2119-29.

[13] Ma Z, Kotaki M, Ramakrishna S. Electrospun cellulose nanofiber as affinity membrane. J Membr Sci 2005; 265: 115-123.

[14] Han SO, Youk JH, Min KD, Kang YO, Park WH. Electrospinning of cellulose acetate nanofibers using a mixed solvent of acetic acid/water: effects of solvent composition on the fiber diameter. Mater Lett 2007; 62(4-5): 759-762.

[15] Cheng NL, Hu NS. Solvents Handbook. Beijing: Chemical Industry Publis; 2008.

[16] He JX, Tang YY, Wang SY. Differences in morphological characteristics of bamboo fibers and other natural cellulose fibers: studies on X-ray diffraction, solid state ${ }^{13} \mathrm{C}-\mathrm{Cp} / \mathrm{MAS} \mathrm{NMR}$, and second derivative FTIR spectroscopy data. Iran Polym J 2007; 16(12): 807818.

[17] Wong SC, Baji A, Leng SW. Effect of fiber diameter on tensile properties of electrospun poly ( $\varepsilon$-caprolactone). Polymer 2008; 49(21): 